\title{
The R-evolution of QCD matrix elements
}

\section{André H. Hoang,}

Max-Planck-Institut für Physik (Werner-Heisenberg-Institut), Föhringer Ring 6,

80805 München, Germany E-mail: ahoang@mppmu . mpg • de

\section{Ambar Jain}

Center for Theoretical Physics, Massachusetts Institute of Technology, Cambridge, MA 02139, USA

E-mail:ambarj@mit.edu

\section{Ignazio Scimemi*}

Departamento de Fìsica Tè̀rica II, Universidad Complutense de Madrid, 28040 Madrid, Spain

E-mail: ignaziosefis.ucm.es

\section{lain W. Stewart}

Center for Theoretical Physics, Massachusetts Institute of Technology, Cambridge, MA 02139 , USA

E-mail:iains@mit.edu

Perturbation series in QCD are generally asymptotic and suffer from so-called infrared renormalon ambiguities. In the context of the standard operator product expansion in $\overline{\mathrm{MS}}$ these ambiguities are compensated by matrix elements of higher dimension operators, but the procedure can be difficult to control due to large numerical cancellations. Explicit subtractions for matrix elements and coefficients, depending on a subtraction scale $R$, can avoid this problem. The appropriate choice for $R$ in the Wilson coefficients can widely vary for different processes. In this talk we discuss renormalization group evolution with the scale $R$, and show that it sums large logarithms in the difference of processes with widely different $R$ 's. We also show that the solution of the $R$-evolution equations can be used to recover the all order asymptotic form of the singularities in the Borel transform of the perturbative series. For the normalization of these singularities we obtain a quickly converging sum rule that only needs the known perturbative coefficients as an input. This sum rule can be used as a novel test for renormalon ambiguities without replying on the large- $\beta_{0}$ approximation.

International Workshop on Effective Field Theories: from the Pion to the Upsilon

1-6 February 2009

Valencia, Spain

\footnotetext{
${ }^{*}$ Speaker.
} 


\section{The $R$-Evolution}

A well known issue in QCD is that perturbative series in $\alpha_{s}$ do not converge, being asymptotic. In perturbative calculations, this feature is caused by contributions from small loop momenta that are factorially enhanced due to running coupling effects. Thus an important task in high order QCD perturbation theory is to establish to which order the perturbative series can be considered "meaningful", or whether it is possible to define subtraction schemes that improve the convergence properties. A common instrument to quantify the high order behavior of perturbative series is the Borel formalism: poles in the Borel transform of the perturbative series in the strong coupling $\alpha_{s}$ correspond to ambiguities that scale like $\Lambda_{\mathrm{QCD}}$ to some integer power $p$. Predictions for physical quantities in perturbative QCD are rendered ambiguity-free in the framework of the operator product expansion (OPE) [1], sometimes formulated within an effective theory (EFT) context. Here renormalon ambiguities of Wilson coefficients are compensated by matrix elements of higher dimensional operators [2]. The most common method to determine poles and the corresponding residues in the Borel transform is based on the computation of massless quark bubble graphs inserted in gluon lines and the "naive non-abelianization approximation" [3], also called the "large- $n_{f}$ " approximation, which seems to work reasonably well in many examples.

In an OPE, short and long wavelength contributions are separated into Wilson coefficients that are computed perturbatively, and matrix elements of operators that require non-perturbative methods. The most common scheme used in the literature for this separation of contributions is the $\overline{\mathrm{MS}}$-scheme. While the $\overline{\mathrm{MS}}$ Wilson coefficients are free from explicit IR divergences to all orders in $\alpha_{s}$, they may still be sensitive to IR physics since they incorporate arbitrarily small loop momenta, leading to renormalon ambiguities. The resulting numerical cancellations between Wilson coefficients and higher dimensional matrix elements are sometimes difficult to control reliably for predictions that require small theoretical uncertainties. This problem can be avoided if schemes for Wilson coefficients and matrix elements with explicit infrared subtractions are employed. In general such subtractions introduce an additional momentum (cutoff) scale, which we denote as $R$. The choice for the scale $R$ depends on the typical distance scale relevant for the quantity described in the OPE expansion. It can therefore differ widely for different applications.

For heavy quark masses such $R$-dependent subtractions schemes have been employed for more than a decade to avoid the $\mathscr{O}\left(\Lambda_{\mathrm{QCD}}\right)$ renormalon ambiguity contained in the pole mass [3] and to define short-distance mass schemes that can be determined with uncertainties smaller than $\Lambda_{\mathrm{QCD}}$. Depending on the process the proper choices for $R$ range from about 1-2 GeV in B-physics or for the description of top jets in the resonance region $[4,5]$, to $15-20 \mathrm{GeV}$ for top pair production at threshold [6], to scales $\gg m$ in cases there the $\overline{\mathrm{MS}}$ mass is the proper mass scheme. For OPE predictions such subtractions have (apart for the just-mentioned heavy quark masses) only become fashionable recently, relevant e.g. for a ambiguity-free definition of the kinetic energy operator $\lambda_{1}$ in the context of HQET [8], or for an ambiguity-free definition of the gap-parameter that governs the first moment of the soft function describing soft radiation in jet production [7].

In this talk we discuss the $R$-evolution of matrix elements in schemes with $R$-dependent subtractions. Much like $\mu$, changes in $R$ are related to changes in renormalization scheme. Due to a power-like dependence on $R$, the solutions are substantially different from the common wellknown logarithmic renormalization group running. We show that the corresponding $R$-RGE sums 
large logarithms contained in the difference of two subtractions involving subtractions scales $R_{0}$ and $R_{1}$ with $R_{0} \ll R_{1}$ [9]. This resolves e.g. the problem of large logs in the relation of low-scale threshold heavy quark masses with $R \ll m_{q}$ and the $\overline{\text { MS}}$-mass with $R \approx m_{q}$. Moreover, taking the formal limit $R_{0} \rightarrow 0$ in the R-RGE solution, we can recover the all order asymptotic behavior of the singularity in the Borel transform that is associated to the subtraction. For the residue of the singularity we obtain a rapidly convergent sum rule that can be determined from the series coefficients of the subtractions without relying on the fermion bubble approximation. This sum rule represents a new method to detect renormalons, and even works for renormalons dominated by non-abelian effects which are hard to detect with fermion bubbles and naive non-abelianization. In this talk we concentrate mostly on the case $p=1$, e.g. relevant for defining heavy quark masses that are free of the $\mathscr{O}\left(\Lambda_{\mathrm{QCD}}\right)$ renormalon. But we emphasize that our method can be applied in a straightforward way for cases with $p>1$ as well. For further details we refer to Refs. [9] and [10].

Consider a low energy non-perturbative matrix element $\theta_{0}$ with mass dimension $p$, which occurs in an OPE for some physical quantity in the $\overline{\mathrm{MS}}$ scheme and has a leading renormalon ambiguity of $\mathscr{O}\left(\Lambda_{\mathrm{QCD}}^{p}\right)$. We can write $\theta_{0}$ as a sum of a matrix element $\theta(R)$ that is free of the $\mathscr{O}\left(\Lambda_{\mathrm{QCD}}^{p}\right)$ renormalon and a perturbation series (the scheme dependent subtraction) $R^{p} \delta \theta(R)$, which contains the $\mathscr{O}\left(\Lambda_{\mathrm{QCD}}^{p}\right)$ renormalon and introduces the subtraction scale $R$. We have

$$
\theta_{0}=\theta(R)+R^{p} \delta \theta(R), \quad \delta \theta(R)=\sum_{n=1}^{\infty} a_{n}\left(\frac{\alpha_{s}(R)}{4 \pi}\right)^{n},
$$

where the $a_{n}$ are constants. Since $\theta_{0}$ is $R$-independent it is straightforward to determine the $R$ evolution equation for the $\theta(R)$, and corresponding $R$-anomalous dimension $\gamma\left[\alpha_{s}(R)\right]$,

$$
R \frac{d}{d R} \theta(R)=-R^{p} \gamma\left[\alpha_{s}(R)\right], \quad \gamma\left[\alpha_{s}(R)\right]=\sum_{n=0}^{\infty} \gamma_{n}\left(\frac{\alpha_{s}(R)}{4 \pi}\right)^{n} .
$$

Here the coefficients are $\gamma_{n-1}=p a_{n}-2 \sum_{k=1}^{n-1} k a_{k} \beta_{n-k-1}$. Since $R d / d R \Lambda_{\mathrm{QCD}}^{p}=0$, the series for $\gamma\left[\alpha_{s}(R)\right]$ is free of the $\mathscr{O}\left(\Lambda_{\mathrm{QCD}}^{p}\right)$ renormalon. We can extend the situation to the more general case where the matrix element is renormalization scale dependent, $\theta_{0}=\theta_{0}(\mu)$. In this case the subtraction is also generally renormalization scale dependent, leading to a matrix element $\theta(\mu, R)$ in an $R$-subtraction scheme. Here $\theta(\mu, R)$ satisfies both a renormalization group equation in $\mu$, and also an $R$-evolution equation for $\mu=R$ that has as a form identical to eqn.(1.2). We note that in the $\mu$-dependent situation, it can be quite subtle to construct a subtraction that leads to consistent evolution equations in $\mu$ and $R$. Examples where this has been achieved are the top quark jet mass that is relevant for describing the single top invariant mass distribution in top production [5], and the gap parameter in the soft function governing dijet production in $e^{+} e^{-}$annihilation [7].

\section{General Solution of the R-RGE}

In order to solve eqn. (1.2) we need an all order solution of the $\alpha_{s}$-RGE. Consider the $\alpha_{s}$-RGE, $R \frac{d \alpha_{s}(R)}{d R}=-\alpha_{s}^{2}(R) /(2 \pi) \sum_{n=0}^{\infty} \beta_{n}\left(\alpha_{s}(R) / 4 \pi\right)^{n}$, where $\beta_{n}$ are $\beta$-function coefficients in an arbitrary scheme for the strong coupling. A solution of this equation is given by

$$
\ln \frac{R_{1}}{R_{0}}=\int_{\alpha_{0}}^{\alpha_{1}} \frac{d \alpha_{R}}{\beta\left[\alpha_{R}\right]}=\int_{t_{1}}^{t_{0}} d t \hat{b}(t)=G\left(t_{0}\right)-G\left(t_{1}\right),
$$


where $\alpha_{i} \equiv \alpha_{s}\left(R_{i}\right), \alpha_{R} \equiv \alpha_{s}(R), t_{i} \equiv-2 \pi /\left(\beta_{0} \alpha_{i}\right)$, and $t \equiv-2 \pi /\left(\beta_{0} \alpha_{R}\right)$. Here

$$
\hat{b}(t)=1+\frac{\hat{b}_{1}}{t}+\frac{\hat{b}_{2}}{t^{2}}+\frac{\hat{b}_{3}}{t^{3}}+\ldots, \quad G(t)=t+\hat{b}_{1} \ln (-t)-\frac{\hat{b}_{2}}{t}-\frac{\hat{b}_{3}}{2 t^{2}}-\ldots,
$$

where for the first few orders $\hat{b}_{1}=\beta_{1} /\left(2 \beta_{0}^{2}\right), \hat{b}_{2}=\left(\beta_{1}^{2}-\beta_{0} \beta_{2}\right) /\left(4 \beta_{0}^{4}\right)$, and $\hat{b}_{3}=\left(\beta_{1}^{3}-2 \beta_{0} \beta_{1} \beta_{2}+\right.$ $\left.\beta_{0}^{2} \beta_{3}\right) /\left(8 \beta_{0}^{6}\right)$. For later convenience we also define $G_{2}(t)=G(t)-t-\hat{b}_{1} \ln (-t)$. From eqn. (2.1) one immediately notes that, $R \exp G(t)=R_{0} \exp G\left(t_{0}\right) \equiv \Lambda_{\mathrm{QCD}}$. This equality demands $R e^{G(t)}$ to be a constant of mass dimension one, which we have defined to be $\Lambda_{\mathrm{QCD}}$ and one can easily check that this definition corresponds to the familiar definition of $\Lambda_{\mathrm{QCD}}^{(k)}$ at $\mathrm{N}^{k} \mathrm{LL}$ order. The solution above is valid for an arbitrary mass-independent scheme for the $\beta$-function, although usually one uses the $\overline{\mathrm{MS}}$ scheme where the $\beta_{i}$ are known to four-loops [12]. Here we present the solution for the $R$-RGE of eqn. (1.2) for $p=1$. For arbitrary $p$, see [10]. Integrating equation (1.2) gives,

$$
\theta\left(R_{1}\right)-\theta\left(R_{0}\right)=-\int_{R_{0}}^{R_{1}} d R \gamma\left[\alpha_{S}(R)\right]=\int_{t_{0}}^{t_{1}} d t \hat{b}(t)\left[\Lambda_{\mathrm{QCD}} e^{-G(t)}\right] \gamma[t],
$$

where we have used the solution of the $\alpha_{s}$-RGE and defined $\gamma[t] \equiv \gamma\left[\alpha_{s}(R)\right]$ using the substitution $t=-2 \pi /\left(\beta_{0} \alpha_{s}(R)\right)$. In order to write the solution in a closed form we define

$$
\begin{gathered}
\hat{b}(t) e^{-G_{2}(t)} \gamma[t] \equiv \sum_{j=0}^{\infty} S_{j}(-t)^{-j-1}, \text { where } S_{0}=\frac{\gamma_{0}}{2 \beta_{0}}, \quad S_{1}=\frac{\gamma_{1}}{\left(2 \beta_{0}\right)^{2}}-\left(\hat{b}_{1}+\hat{b}_{2}\right) \frac{\gamma_{0}}{2 \beta_{0}}, \\
S_{2}=\frac{\gamma_{2}}{\left(2 \beta_{0}\right)^{3}}-\left(\hat{b}_{1}+\hat{b}_{2}\right) \frac{\gamma_{1}}{\left(2 \beta_{0}\right)^{2}}+\left[\left(1+\hat{b}_{1}\right) \hat{b}_{2}+\left(\hat{b}_{2}^{2}+\hat{b}_{3}\right) / 2\right] \frac{\gamma_{0}}{\left(2 \beta_{0}\right)}, \ldots
\end{gathered}
$$

Now substituting eqn. (2.4) into eqn. (2.3) and using the definition of the incomplete gamma function with the standard convention of integration above the cut, $\int_{t}^{\infty} d t^{\prime}\left(-t^{\prime}\right)^{c-1} e^{-t^{\prime}}=-e^{-i \pi c} \Gamma(c, t)$, we get the $\mathrm{N}^{k} \mathrm{LL}$ solution that is given by

$$
\left[\theta\left(R_{1}\right)-\theta\left(R_{0}\right)\right]^{\mathrm{N}^{k} \mathrm{LL}}=\left(\Lambda_{\mathrm{QCD}}^{(k)}\right) \sum_{j=0}^{k} S_{j}(-1)^{j} e^{i \pi \hat{b}_{1}}\left[\Gamma\left(-j-\hat{b}_{1}, t_{1}\right)-\Gamma\left(-j-\hat{b}_{1}, t_{0}\right)\right] .
$$

Here $\Lambda_{\mathrm{QCD}}^{(k)}$ is the $\mathrm{N}^{k} \mathrm{LL}$ solution of: $R \exp G(t) \equiv \Lambda_{\mathrm{QCD}}$. The integrand in eqn. (2.3) has an essential singularity and a branch point at $t=0$ due to infinitely many negative powers of $t$ and a branch cut on the positive real axis due to the $(-t)^{-\hat{b}_{1}}$. For $R_{0}, R_{1}>\Lambda_{\mathrm{QCD}}$ we have $t_{0}, t_{1}<0$, so the range of integration is away from the branch cut and the integral is well defined. To write a closed form expression we have split the integral into a difference of two incomplete gamma functions, and with the $e^{i \pi \hat{b}_{1}}$ factor this solution is real. Note that only the difference of the gamma functions appearing in eq. (2.5) is independent of the convention for treating the cut.

To see what kind of perturbative terms the solution in eq. (2.5) contains, we take the $k=0$ case (LL) and recall the asymptotic expansion for $t \rightarrow-\infty, \Gamma[c, t] \stackrel{\text { asym }}{=} e^{-t} t^{c-1} \sum_{n=0}^{\infty} \frac{\Gamma(1-c+n)}{(-t)^{n} \Gamma(1-c)}$. For $c=0$ this yields $\Lambda_{\mathrm{QCD}}^{(0)} \Gamma[0, t] \stackrel{\text { asym }}{=}-2 R \sum_{n=0}^{\infty} 2^{n} n !\left(\beta_{0} \alpha_{s}(R) /(4 \pi)\right)^{n+1}$, where we used the LL relation $\Lambda_{\mathrm{QCD}}^{(0)} e^{-t}=R$. This is a divergent series, but for the LL solution of the RGE

$$
\begin{aligned}
{\left[\theta\left(R_{1}\right)-\theta\left(R_{0}\right)\right]^{\mathrm{LL}} } & =\frac{-\gamma_{0} R_{1}}{2 \beta_{0}} \sum_{n=0}^{\infty}\left[\frac{\beta_{0} \alpha_{1}}{2 \pi}\right]^{n+1} n !\left(1-\frac{R_{0}}{R_{1}} \sum_{k=0}^{n} \frac{1}{k !} \ln ^{k} \frac{R_{1}}{R_{0}}\right) \\
& =-\frac{\gamma_{0} R_{0}}{2 \beta_{0}} \sum_{n=0}^{\infty}\left[\frac{\beta_{0} \alpha_{1}}{2 \pi}\right]^{n+1} \sum_{k=n+1}^{\infty} \frac{n !}{k !} \ln ^{k} \frac{R_{1}}{R_{0}},
\end{aligned}
$$


which is convergent since $\beta_{0} \alpha_{s}\left(R_{1}\right) \ln \left(R_{1} / R_{0}\right) /(2 \pi)<1$. Eqn. (2.6) displays the problem of large $\log$ in fixed order perturbation theory for $R_{1} \gg R_{0}$. The RGE in $R$ encodes IR physics from the large order behavior of perturbation theory. It causes a rearrangement of the IR fluctuations included in $\theta(R)$ in going from $R_{0}$ to $R_{1}$ without reintroducing a renormalon.

\section{The R-RGE and the Leading Renormalon Ambiguity}

In this section we demonstrate a novel application of the $R$-RGE by deriving the leading renormalon ambiguity of $\theta_{0}$ of eqn. (1.1) in the Borel plane and providing a sum rule to perturbatively determine the normalization of the renormalon ambiguity. A sum rule of this kind for $u=1 / 2$ ambiguity of the pole mass was first provided in our earlier work [9].

We note ${ }^{1}$ that in the complex $R$-plane away from the positive real axis, $\lim _{R \rightarrow 0} \alpha_{s}(R)=0$. Therefore $\delta \theta(R)$ of eqn. (1.1) vanishes at $R=0$ and we get $\lim _{R \rightarrow 0} \theta(R)=\theta_{0}$. It is important to note that though $\alpha_{s}(R=0)$ is single-valued, but the process of taking the limit $R \rightarrow 0$ is not unambiguous due to the branch cut on $0<R<\Lambda_{\mathrm{QCD}}$. It is the limiting procedure that reintroduces the ambiguity. Thus, taking limit $R_{0} \rightarrow 0$ in eqn. (1.1) we get

$$
\theta\left(R_{1}\right)-\theta_{0}=-\Lambda_{\mathrm{QCD}} \sum_{j=0}^{\infty} S_{j} \int_{t_{1}}^{\infty} d t \frac{e^{-t}}{(-t)^{j+\hat{b}_{1}+1}}
$$

The LHS has a renormalon ambiguity in $\theta_{0}$, which on the RHS is in the integral, the integrand has a branch cut on the positive real axis in the complex $t$-plane, and the integration must be performed by either going above the positive real axis or below it. Using the asymptotic expansion for this integral, which is the same as that for $\Gamma(c, t)$, and expanding $e^{G_{2}(t)}=\sum_{\ell=0}^{\infty} \frac{g_{\ell}}{(-t)^{\ell}}$, we get a power series expansion in $1 /(-t)=\beta_{0} \alpha_{s}(R) /(2 \pi)$,

$$
\theta(R)-\theta_{0}=-R \sum_{j, n, \ell=0}^{\infty} S_{j} g_{\ell} \frac{\Gamma\left(1+j+n+\hat{b}_{1}\right)}{\Gamma\left(1+j+\hat{b}_{1}\right)(-t)^{n+j+\ell+1}}
$$

On taking the Borel transform ${ }^{2}$, after some algebra, we get

$$
B(u)=2 R\left[\sum_{\ell=0}^{\infty} g_{\ell} Q_{\ell}(u)-P_{1 / 2} \sum_{\ell=0}^{\infty} g_{\ell} \frac{\Gamma\left(1+\hat{b}_{1}-\ell\right)}{(1-2 u)^{1+\hat{b}_{1}-\ell}}\right],
$$

where

$$
P_{1 / 2}=\lim _{k \rightarrow \infty} P_{1 / 2}^{N^{k} L L}=\lim _{k \rightarrow \infty} \sum_{j=0}^{k} \frac{S_{j}}{\Gamma\left(1+\hat{b}_{1}+j\right)}
$$

and $Q(u)$ is a function convergent in a finite neighborhood of $u=1 / 2$. The difference, $\theta(R)-$ $\theta_{0}$, can be obtained by the inverse Borel transform, $\theta(R)-\theta_{0}=\int_{0}^{\infty} d u B(u) e^{-u 4 \pi /\left(\beta_{0} \alpha_{s}(R)\right)}$. This

\footnotetext{
${ }^{1}$ Eqn. (2.1) has the solution that as $t$ goes to infinity, $R \rightarrow 0$ with a phase $e^{ \pm i \pi \hat{b}_{1}}$. That is to say $R$ cannot approach 0 from the positive real axis. $R$ must go to 0 either from above the positive real axis or below it. This is so because there is a cut on the positive real axis for $0<R<\Lambda_{\mathrm{QCD}}$. This cut corresponds to the branch cut in the $t$-plane for the integrand of the equation (2.3). Nevertheless, $t$ as a function of $R$ is single-valued at $R=0$, corresponding to $\alpha_{s}(R=0)=0$.

${ }^{2}$ Taking the Borel transform amounts to making the replacement $1 / t^{n+1} \rightarrow 2(2 u)^{n} / \Gamma(n+1)$. In our convention, which is also the most widely used, $u$ is Borel conjugate of $\beta_{0} \alpha_{s} /(4 \pi)$.
} 
integral is ambiguous because eqn. (3.2) has a branch cut for $u>1 / 2$. Thus we have obtained, in eqn. (3.2), the structure of the leading renormalon ambiguity in $\theta_{0}$, represented by the second term on the RHS, along with its normalization $P_{1 / 2}$. Here $P_{1 / 2}$ is an absolutely convergent infinite series [9] constructed from the coefficients $S_{j}$ that appear in the solution of the R-RGE (eqn. (2.4)). The $S_{j}$ are in turn determined by the $R$-anomalous dimension coefficients $\gamma_{n}$ (eqn. (1.2)). Therefore $P_{1 / 2}^{\mathrm{N}^{k} L \mathrm{~L}}$ is perturbatively determinable in terms of the original coefficients $a_{n}$ of $\delta \theta(R)$.

For any given $\theta(R)$ the process described above can be repeated with a rescaling $R \rightarrow \lambda R$. This does not change the normalization of the renormalon ambiguity, since it amounts to only changing the scale $R$, or equivalently changing the scheme. In this new $\lambda$-dependent scheme, we have $\lambda$-dependent $S_{j}$, where the first few are given by

$$
\begin{aligned}
& S_{0}(\lambda)=\lambda S_{0}, \quad S_{1}(\lambda)=\lambda S_{1}-\frac{a_{1} \lambda \log (\lambda)}{2 \beta_{0}}, \\
& S_{2}(\lambda)=\lambda S_{2}+\frac{\lambda \log (\lambda)\left(-a_{2}+a_{1}\left(\hat{b}_{2}+2\right) \beta_{0}+a_{1} \beta_{0} \log (\lambda)\right)}{2 \beta_{0}^{2}} .
\end{aligned}
$$

As a result $P_{1 / 2}^{\mathrm{N}^{\mathrm{k}} \mathrm{LL}}$ depends on $\lambda$, but $P_{1 / 2}$ will be $\lambda$-independent. This gives a way to assign errors and test for convergence of the partial sum $P_{1 / 2}^{\mathrm{N}^{k} \mathrm{LL}}$. Distinguishing $P_{1 / 2} \neq 0$ and $P_{1 / 2}=0$ provides a test for the presence of a renormalon ambiguity. We propose to keep $0.5<\lambda<2$, so that the perturbative expansion is well behaved.

\section{Applications of the Renormalon Sum Rule}

As an application of the sum rule, we calculate the residue of the renormalon ambiguity of the heavy quark pole mass. Using eqn. (3.3) along with $S_{j}$ 's from eqn. (3.4), we obtain $P_{1 / 2}$ as a function of scaling $\lambda$ in MSR $[9,11,10]$, static $[9,10]$ and PS [3] mass schemes up to NNLL order. This is shown in Figure 1, where the band that envelops $P_{1 / 2}$ curves for these schemes (with $n_{f}=5$ light flavors) is plotted at LL, NLL and NNLL order. Since the pole mass renormalon ambiguity is scheme independent, we expect the sum rule to converge to a scheme independent number. This is clearly seen from the figure, since the band at the NNLL order is significantly narrower with weak $\lambda$-dependence. From the narrow red (NNLL) band we estimate that $P_{1 / 2}^{\text {mass }}=0.47 \pm 0.10$, which is the normalization of the order $\Lambda_{\mathrm{QCD}}$ renormalon ambiguity in the heavy quark pole mass. For comparison, the bubble chain approximation gives $P_{1 / 2}^{\text {mass }}=0.80$, overestimating by a factor of 2 . Our values are consistent with the determination of the normalization of the renormalon ambiguity in Ref. [14].

The Wilson coefficient $C_{\mathrm{cm}}\left(m_{Q}, \mu\right)$ of the chromomagnetic HQET operator $\bar{h}_{v} \sigma \cdot G h_{v}$, is known to have a $\mathscr{O}\left(\Lambda_{\mathrm{QCD}} / m_{Q}\right)$ renormalon. We will test for this renormalon using our renormalon sum rule and determine its size. $C_{\mathrm{cm}}$ is known to three loops [13] and at $\mu=m_{Q}$, it is given by, $C_{\mathrm{cm}}\left(m_{Q}, m_{Q}\right)-1=\sum_{i=1}^{\infty} a_{i}\left(\frac{\alpha_{s}\left(m_{Q}\right)}{4 \pi}\right)^{i}$. We are looking for $n !\left(2 \beta_{0}\right)^{n}$ growth $(p=1)$ in the coefficients of $\alpha_{s}(R)^{n}\left(m_{Q}\right)$. To test this, we take the known $\overline{\mathrm{MS}}$ scheme results $a_{1}=8.66703, a_{2}=$ $\left(350.347-30.6037 n_{f}\right), a_{3}=\left(21985.2-3470.72 n_{f}+101.8 n_{f}^{2}\right)[13]$ and substitute in eqn. (3.4), which we then use in eqn. (3.3) to get the $P_{1 / 2}$ sum rule as a function of $\lambda$. This sum rule is plotted in Figure 1 for $n_{f}=3$ (thin lines) and $n_{f}=4$ (thick lines) light flavors at the LL(dotted), 

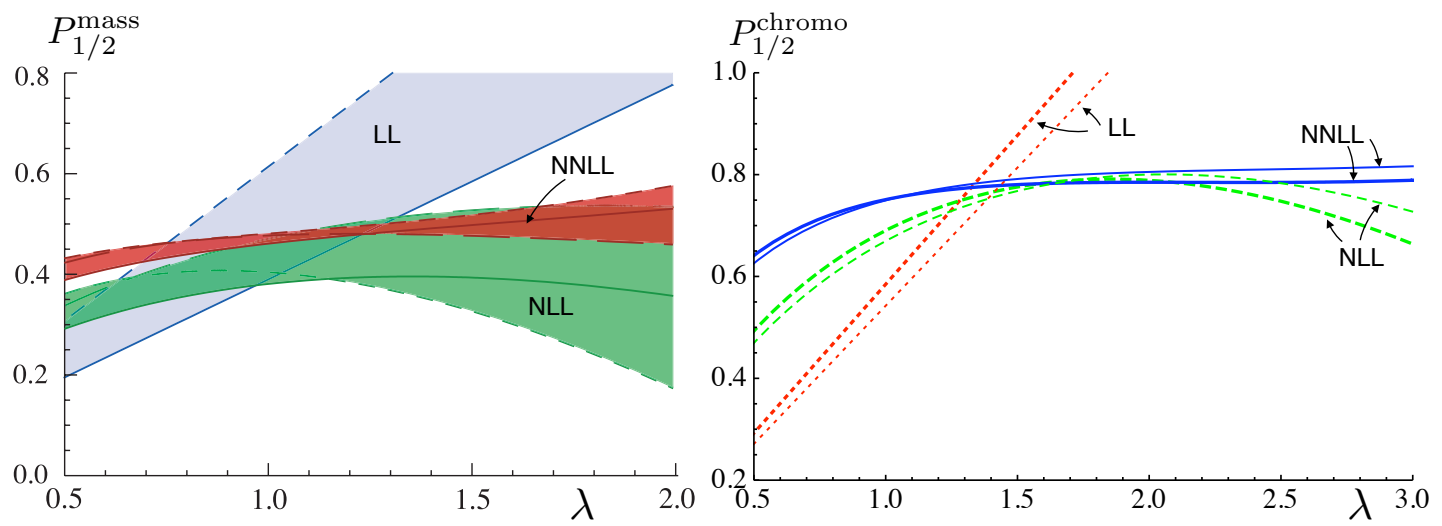

Figure 1: The $P_{1 / 2}$ sum rule for the heavy quark pole mass (left) and the chromomagnetic operator Wilson coefficient (right).

NLL(dashed) and NNLL(solid) orders; convergence is quite evident and from the $\lambda$ dependence we estimate $P_{1 / 2}^{\text {chromo }}=0.72 \pm 0.09\left(n_{f}=3\right)$, and $P_{1 / 2}^{\text {chromo }}=0.71 \pm 0.07\left(n_{f}=4\right)$. The sum rule yields a clear renormalon. For further details and discussions regarding concepts presented in this proceeding, including some direct applications of the $R$-RGE, see $[9,10]$.

Acknowledgments: This work was supported by the EU network contract MRTN-CT-2006035482 (Flavianet), and the Office of Nuclear Physics of the U.S. Department of Energy, contract DE-FG02-94ER40818.

\section{References}

[1] A. H. Mueller, Nucl. Phys. B 250, 327 (1985).

[2] M. E. Luke, A. V. Manohar and M. J. Savage, Phys. Rev. D 51, 4924 (1995) [arXiv:hep-ph/9407407].

[3] M. Beneke, Phys. Rept. 317, 1 (1999).

[4] S. Fleming, A. H. Hoang, S. Mantry and I. W. Stewart, Phys. Rev. D 77, 074010 (2008) [arXiv:hep-ph/0703207].

[5] A. Jain, I. Scimemi and I. W. Stewart, Phys. Rev. D 77, 094008 (2008) [arXiv:0801.0743 [hep-ph]].

[6] A. H. Hoang et al., Eur. Phys. J. direct C 2, 1 (2000) [arXiv:hep-ph/0001286].

[7] A. H. Hoang and S. Kluth, arXiv:0806.3852 [hep-ph].

[8] I. I. Y. Bigi, M. A. Shifman, N. G. Uraltsev and A. I. Vainshtein, Phys. Rev. D 52 (1995) 196.

[9] A. H. Hoang, A. Jain, I. Scimemi and I. W. Stewart, Phys. Rev. Lett. 101, 151602 (2008) [arXiv:0803.4214 [hep-ph]].

[10] A. H. Hoang, A. Jain, I. Scimemi and I. W. Stewart, work in progress.

[11] A. H. Hoang and I. W. Stewart, Nucl. Phys. Proc. Suppl. 185, 220 (2008) [arXiv:0808.0222 [hep-ph]].

[12] T. van Ritbergen, J. A. M. Vermaseren and S. A. Larin, Phys. Lett. B 400 (1997) 379.

[13] A. G. Grozin and M. Neubert, Nucl. Phys. B 508, 311 (1997) ; A. G. Grozin, P. Marquard, J. H. Piclum and M. Steinhauser, Nucl. Phys. B 789, 277 (2008)

[14] A. Pineda, JHEP 0106, 022 (2001). 